\title{
Spatial contrast sensitivity and the diagnosis of amblyopia
}

\author{
A C W VOLKERS, ${ }^{1} \mathrm{~K}$ H HAGEMANS, ${ }^{2} \mathrm{G}$ J VAN DER WILDT, ${ }^{1}$ AND \\ P I M SCHMITZ
}

From the 'Department of Biomedical Physics and Technology, Erasmus University, PO Box 1738, NL-3000 DR Rotterdam; ${ }^{2}$ Health Centre, De Linie 2, Capelle a.d. IJssel; and the ${ }^{3}$ Institute of Biostatistics, Erasmus University, Rotterdam, The Netherlands

SUMMARY The relationship was studied between spatial contrast sensitivity function and type of pathology of the visual system. Two characteristics were found to be typical for amblyopia: (1) there is a discrepancy between the high-frequency cut-off ('grating acuity') and the Snellen acuity; (2) the contrast sensitivity is strongly dependent on the width of the stimulus. In optic nerve degeneration a decrease in contrast sensitivity is found at low spatial frequencies. Decreased contrast sensitivity at high spatial frequencies is atypical but occurs in those disorders associated with decreased Snellen visual acuity. From these findings a relationship is defined which enables spatial contrast sensitivity tests to be used to differentiate between amblyopia and optic nerve degeneration.

The ophthalmologist is faced with the task of diagnosing the cause of any visual impediment. After optimal correction and examination by slit-lamp or ophthalmoscope there remain some patients in whom the diagnosis is not clear. Their visual loss may be due to optic nerve degeneration or to amblyopia ('lazy eye'). It would be helpful if a simple method were available to distinguish between these two possibilities. Determination of spatial contrast sensitivity function (CSF) might be such a relatively simple method.

The Snellen optotype chart is a measure of the minimum angle of resolution at high contrast. In CSF the maximum discernible spatial frequency can also be interpreted as a measure of the resolving power: the 'grating acuity' can be calculated from this frequency. Although the stimuli with which the visual acuity and the grating acuity are determined are quite different (optotypes and line patterns respectively), it is interesting to compare the visual acuity and the grating acuity because both values are measures of the spatial resolution at high contrast. The CSF provides more information than the Snellen acuity, while it also estimates the spatial resolution at low

Correspondence to A C W Volkers, Erasmus Universiteit Rotterdam, Postbus 1738, 3000 DR Rotterdam, The Netherlands. contrast or, in other words, how well coarser structures can be seen at low contrast.'

The study of contrast sensitivity function as it is related to several types of pathology is not new. Various workers have reported on contrast sensitivity in amblyopia ${ }^{1-9}$ and in optic nerve disorders. ${ }^{45}$ The CSF has been described in optic nerve lesions ${ }^{10-12}$ and in cataract. ${ }^{13}$ Most of these publications deal with rather small numbers of cases, and almost all concern our understanding of specific disorders of the visual system, but there are no reports on the evaluation of CSF as a diagnostic aid in neuro-ophthalmological practice.

The aim of this study was threefold: first, to determine to what extent the shape of the CSF is related to the particular type of pathology; secondly, to evaluate the effectiveness of spatial contrast sensitivity tests for the diagnosis of amblyopia, especially with regard to the differentiation between amblyopia and optic nerve degeneration with decreased visual acuity; and thirdly to compare the visual function in optic nerve degeneration combined with normal visual acuity with visual function in a group of normal eyes.

In a pilot study by Hagemans and Van der Wildt ${ }^{9}$ in a small group of patients it was found that the 
dependence of contrast sensitivity on the width of the stimulus was much greater for amblyopes than for normal subjects or patients with other pathology.

\section{Subjects and methods}

The subjects who took part in this study were chosen from among the outpatients of an ophthalmological practice. They were divided into several groups according to the type of pathology, thus: amblyopia $(n=98)$, optic nerve degeneration $(n=42)$, and cataract $(n=10)$. A group of normal persons $(n=26)$, that is, without any known pathology or complaints, were also examined.

The group of patients with optic nerve degeneration comprised those who had suffered from optic neuritis in the previous three years, with or without other evidence of multiple sclerosis. Optic neuritis was electrophysiologically diagnosed in that period. This group was divided into two subgroups, those with normal visual acuities (greater than or equal to $1.0 ; n=16)$ and those with diminished visual acuities (less than 1.0;n=26). The group of cataractous eyes was examined to enable a comparison to be made between groups of eyes with decreased visual acuity originating from different causes.

Cases with evidence of multiple pathology were excluded. From a statistical point of view each patient was allowed to occur only once in the series. Hence in cases of binocular disease only one eye (chosen at random) was used. Five amblyopes had binocular disease, while 93 had monocular. The numbers of binocular and monocular cases of optic nerve degeneration were 7 and 35 , and of cataract 4 and 6 , respectively.

The mean age was 35 years, standard deviation 10 years, for both normal and amblyopic subjects; 45 , SD 17, years for subjects in both optic nerve degeneration subgroups; and 59, SD 11, years for the subjects in the cataract group.

The visual acuity of all subjects was determined by the Snellen optotype chart.

\section{DETERMINATION OF CONTRAST SENSITIVITY}

The contrast sensitivity (CS) was determined by presenting vertical sinusoidal gratings on a television monitor. The contrast of the stimulus was variable; the contrast threshold was determined for each eye separately by a modified von Békésy tracking technique. ${ }^{14}$ The spatial frequency of the grating and the width of the stimulus could be adjusted. The height of the stimulus always subtended $5^{\circ}$ visual angle; the average luminance of the stimulus was $5 \mathrm{~cd} / \mathrm{m}^{2}$. The examinations were performed in a dark room. The grating was counterphased every $0.6 \mathrm{~s}$ to prevent after-images.
The CSF was determined for spatial frequencies ranging from $0 \cdot 1$ to 25.6 cycles per degree (c/deg) in steps of a factor 2 . Up to $0.4 \mathrm{c} / \mathrm{deg}$ the viewing distance was $50 \mathrm{~cm}$, resulting in a stimulus width of $32^{\circ}$; from 0.8 to $25.6 \mathrm{c} / \mathrm{deg}$ the viewing distance was $200 \mathrm{~cm}$, which gave a width of $8^{\circ}$. For lower frequencies the stimulus had to be wider, because if there are fewer than 5 cycles within the stimulus the number of cycles has an influence on the CS. ${ }^{15-18}$ The CS is defined as the reciprocal of the value of the contrast at the threshold.

When the CS was determined as a function of the width of the stimulus, the monitor was at a viewing distance of $100 \mathrm{~cm}$; the width of the stimulus was changed from $16^{\circ}$ to $1^{\circ}$ in steps of a factor 0.5 . The width dependence was determined at a spatial frequency of $1.6 \mathrm{c} / \mathrm{deg}$.

The CS data were automatically plotted in double logarithmic form against spatial frequency or against the width of the stimulus, depending on the type of determination. The apparatus with which the measurements were performed was specially designed for clinical use. ${ }^{19}$ The measuring procedure runs automatically and is controlled by a microprocessor system. The whole measuring sequence including patient instruction takes about 25 minutes for both eyes.

For each of the CSFs the corresponding grating acuity was calculated by extrapolating the high frequency part of the CSF to the maximum contrast $(C S=1)$. A quadratic function was used for the extrapolation, as this type of curve yielded a good fit for the appropriate part of the CSF. The grating acuity is expressed as the reciprocal of the minimum angle of resolution in minutes of arc, as is the Snellen visual acuity. The acuity $A(1 / \mathrm{min}$ of arc) is equivalent to a spatial frequency of $30 \times \mathrm{A}$ [c/deg].

The slope of the CS between $1^{\circ}$ and $4^{\circ}$ width was taken as the measure for the width dependence of the CS. This was expressed as:

$$
S=\left(\log \left(\operatorname{CS}\left(4^{\circ}\right)\right)-\log \left(\operatorname{CS}\left(1^{\circ}\right)\right) / \log (4) .\right.
$$

\section{Results}

Point by point averaging of the data points of the CSFs within each of the groups yielded the groupspecific CSFs. The curves for the group of normal eyes and for the group of amblyopic eyes are depicted in Fig. 1. The mean visual acuity for the group of normal eyes was 1.34 ; the mean grating acuity was $1 \cdot 18$.

As is to be expected from the lower mean visual acuity in the amblyopic eyes $(0 \cdot 39)$, contrast sensitivity at the higher spatial frequencies (CS(HF)) was low. This was associated with a decrease in the grating acuity, which had a mean value of $0 \cdot 80$. 
Fig. 1 Lower left: Averaged CSFs for 26 normal visual systems and for 98 amblyopic eyes. The dashed lines at the high frequency end of the CSFs indicate extrapolation to the grating acuity. Lower right: The relationships between contrast sensitivity and width of the stimulus ('width dependence') for normal eyes and for amblyopic eyes. Upper left and right: Ratio of the two curves in the lower part with the normal one as the reference. The curve on the left (the ratio of the two CSFs) is called the 'visuogram'.
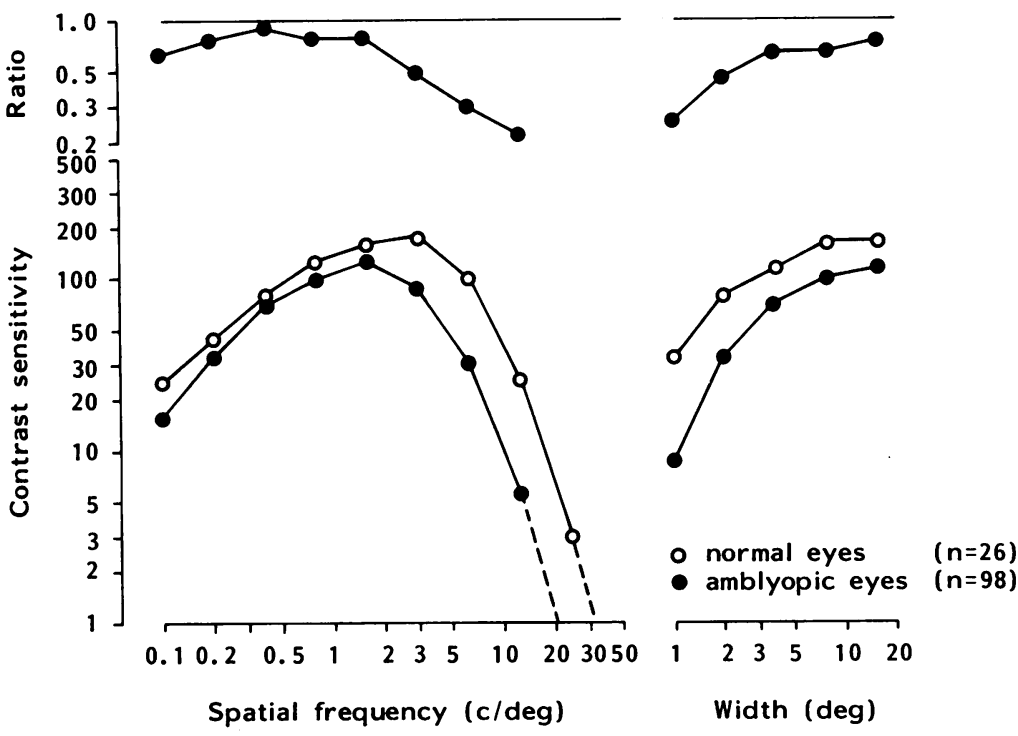

Hence the resolving power calculated from the CSF was about twice the value determined with the Snellen letter chart. The graph at the top of Fig. 1 shows the ratio of the value for amblyopic eyes to that for normal eyes. This type of curve is called a "visuogram. ${ }^{20}$ The difference between amblyopic and normal eyes became more pronounced at higher frequencies.

The right-hand graphs show the relationship between CS and the width of the stimulus. Below a width of $4^{\circ}$ the CS decreased considerably, which means that for a narrower grating presentation more

contrast is needed to reach the threshold. In the amblyopia group the CS decreased more rapidly as the width of the grating was reduced than in the normal group: the slope between $1^{\circ}$ and $4^{\circ}$ was much greater in the amblyopic visual systems. This result, which was anticipated in the pilot study performed by Hagemans and van der Wildt, ${ }^{9}$ was confirmed by this study of 98 amblyopic eyes. The results were almost identical among subdivisions of the group of amblyopic eyes: 41 cases of anisometropia, 32 cases of strabismus, and 25 cases of unknown origin. These results are not shown here.

Fig. 2. Lower left: Averaged CSFs for 26 normal eyes, for optic nerve degeneration with normal visual acuity $(n=16)$, and with decreased visual acuity $(n=26)$. Lower right: Width dependence for the same groups. Upper: Ratio curves with the normal curve as the reference.

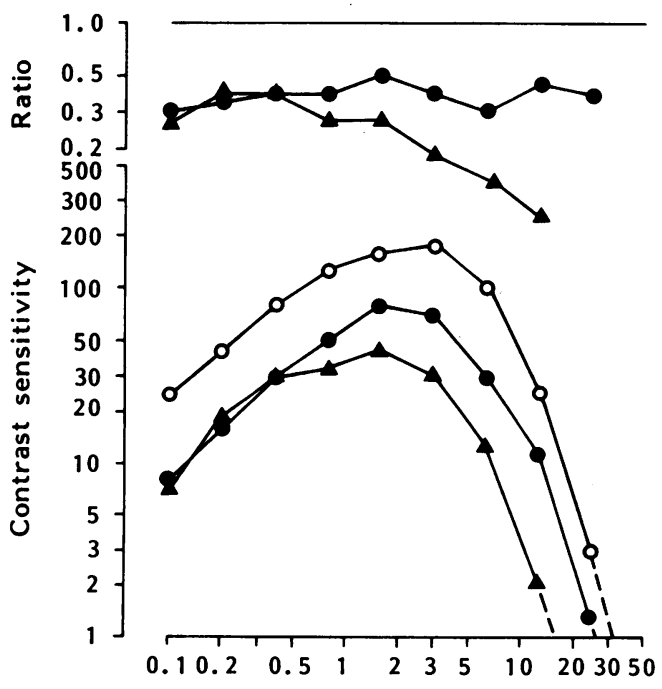

Spatial frequency (c/deg) 


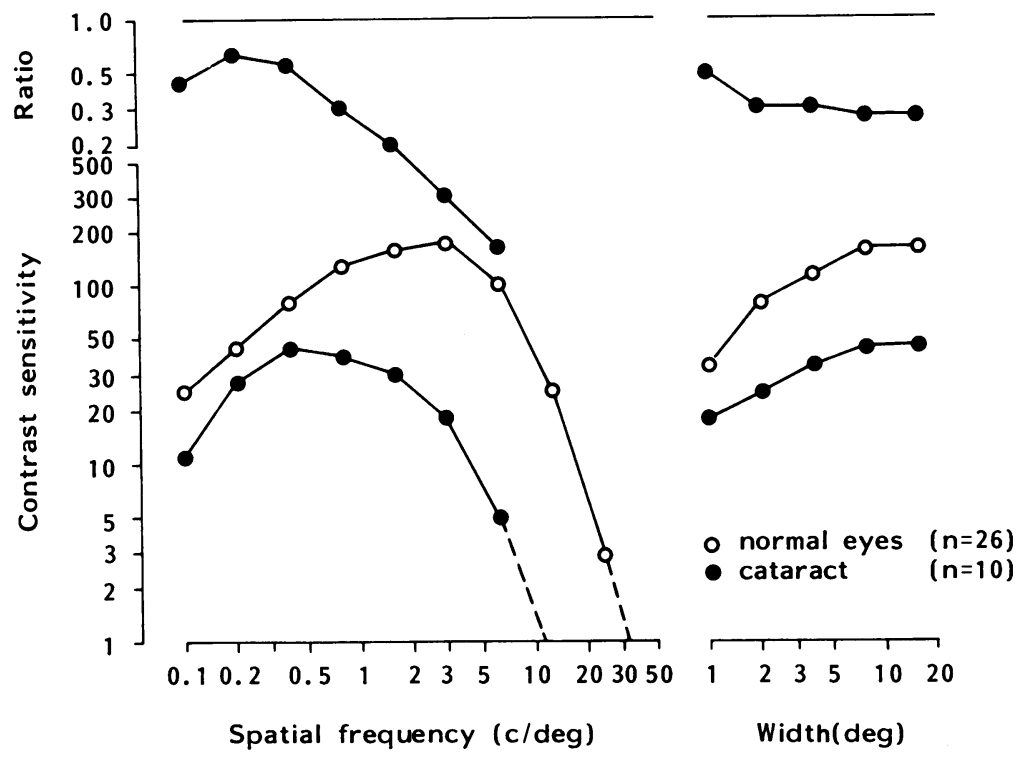

Fig. 3 Lower left: Averaged CSFs for 26 normal and 10 cataractous eyes. Lower right: Width dependence for the same groups. Upper: Ratio curves with the normal curve as the reference.

The averaged results for the two subgroups of eyes with optic nerve degeneration are shown in Fig. 2; the CSF for the normal group is given as a reference. The CSs for both optic nerve degeneration subgroups were lower than for the normal group at all spatial frequencies. For both subgroups the CS in the low spatial frequency range (CS(LF)) was reduced by about the same amount, while the CS(HF) was lower in the low vision subgroup than in the subgroup with normal visual acuity. The calculated grating acuity values were 0.71 and 1.01 respectively. The corresponding visual acuities were 0.49 and 1.14 . In both subgroups the width dependence (see right-hand graph) had a similar form to that in the reference group, but the whole curve was lower. This is to be expected from the lower CS value at $1.6 \mathrm{c} / \mathrm{deg}$ in the CSF. The results were almost the same among subgroups containing 23 cases of optic neuritis without and 19 cases of optic neuritis with multiple sclerosis. This is not shown here.

Comparison of the averaged results for the cataractous eyes with those of the normal group in Fig. 3 indicates a reduction in CS(HF). This was expected from the lower mean visual acuity in this group $(0.45)$; the grating acuity $(0.48)$ was in accordance with this.

On the basis of these results we can recognise at least three differences between some of the groups. These differences, which we shall study in further detail, can be seen in: (1) the discrepancy between the two types of spatial resolution estimate-visual acuity and grating acuity; (2) the dependence of the CS on the width of the stimulus; (3) the contrast sensitivity for the low spatial frequency range.

\section{Discussion}

\section{VISUAL ACUITY AND GRATING ACUITY}

The Snellen test of visual acuity (VA) is an important tool in ophthalmological practice. It is a measure of the performance of the visual system at high contrast, and it is claimed that it evaluates the patient's reading ability. It is expressed as the reciprocal of the minimum angle of resolution in minutes of arc. The grating acuity (GA) may also be expressed in the same units; half a cycle of the highest visible frequency is taken as the minimum angle of resolution. Some relationship between VA and GA may be expected, though the stimuli are different (respectively optotypes and gratings).

Our results indicate that there is a discrepancy between the two estimates for the spatial resolution. For the normal group and for the optic nerve degeneration subgroup with normal VA the VA is on average greater than the GA, while for the group of amblyopic eyes the VA is much lower than the GA. The VA is also lower than the GA for the low vision subgroup with optic nerve degeneration, albeit to a lesser extent. Only for the group of cataractous eyes are VA and GA nearly equal.

In this study it is not proved whether in amblyopia the GA is an overestimation of the real spatial resolution or the VA an underestimation. Levi and colleagues found identical differences ${ }^{212}$ and suggest it is because amblyopic visual systems have edge sensitive mechanisms broader than normal. ${ }^{23}{ }^{24}$ In five amblyopic subjects we also determined the VA with single letters (Sheridan-Gardiner test), and in four of them this measure for VA coincided with the GA, 

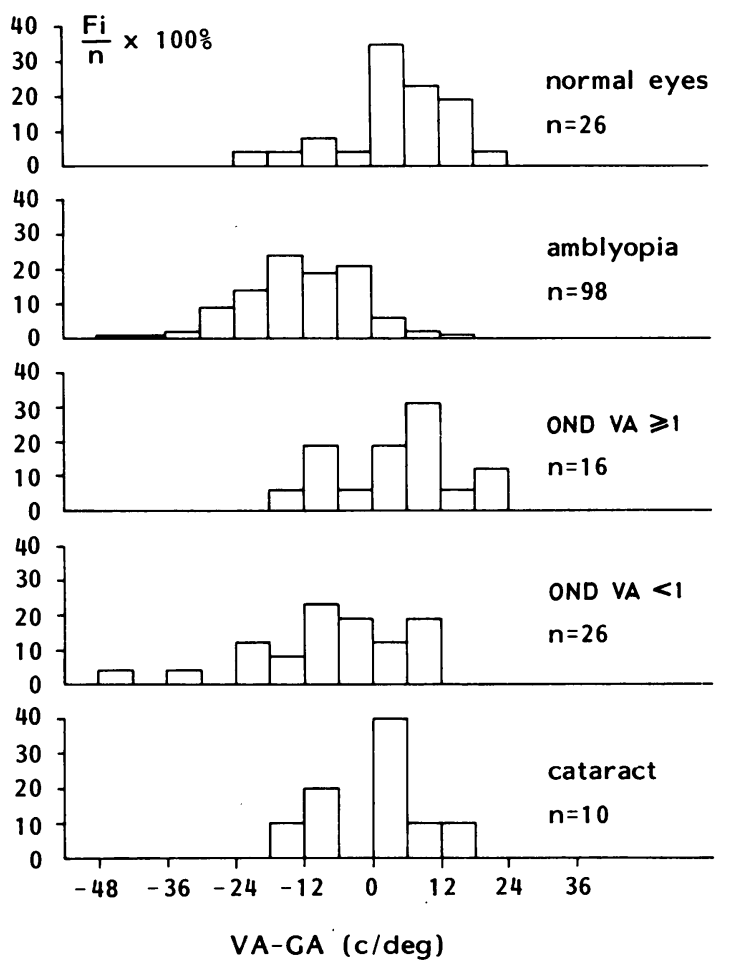

Fig. 4 Frequency distribution of difference between visual acuity and grating acuity in the groups of normal, amblyopic, optic nerve degenerative (normal and low visual acuity), and cataractous eyes. The distributions are normalised to a total of $100 \%$.

though the values were still subnormal. This so called crowding phenomenon or separation difficulty ${ }^{25}{ }^{26} \mathrm{can}$ be explained by the same edge sensitive mechanism.

The discrepancy between VA and GA (which is greatest in the amblyopia group) was further studied for its potential as a diagnostic measure. The discrepancy was expressed as the difference between VA and GA. Fig. 4 gives the frequency distributions of VA-GA for the groups of normal eyes and amblyopic eyes, for both subgroups of eyes with optic nerve degeneration, and for the group of cataractous eyes. Each distribution is normalised to a total of $100 \%$. An analysis of variance ${ }^{27}$ showed that the distributions cannot be regarded as samples from the same population: $F(4,175)=29 \cdot 7 ; p<0.0001$. These results suggest that the VA-GA difference can be used to differentiate between some of the groups. By means of Tukey's multiple range test,${ }^{28}$ using $\alpha=0.05$, it was found that the amblyopic group of eyes differs significantly from all the other groups of eyes except the low vision subgroup with optic nerve degeneration.
WIDTH DEPENDENCE

The second differentiating finding is the dependence of CS on the width of the stimulus. To detect a narrower grating more contrast is needed. In our experimental data the largest effect was found between $1^{\circ}$ and $2^{\circ}$ width. To obtain a statistical spread the slope $S$ was calculated for the range from $1^{\circ}$ to $4^{\circ}$. The slope is much higher for amblyopic eyes than for non-amblyopic eyes, as shown in Figs. 1 to 3.

An explanation for differences in width dependence between amblyopic and other eyes may be found in an influence of the sharp luminance discontinuity on the contrast needed for perception of the grating when the edges of the narrowing stimulus approach each other. It is not known whether the edge sensitive mechanism of Levi et al..$^{24}$ is sufficient as an explanation for the higher slope $\mathrm{S}$ in amblyopia, as the former seemed to play a part at approximately $10 \mathrm{~min}$ of arc, while the effect in the latter already happens at a half width of 120 min of arc. If an edge sensitive mechanism explains differences in VA-GA as well in width dependence among several groups of eyes, then there must be a correlation between the two variables. Indeed for the groups of 26 normal and 98 amblyopic eyes there is a significant correlation between VA-GA and the slope S (using $\alpha=0 \cdot 05)$, though the association is weak $(r=-0 \cdot 39)$. Research in this field is continuing. ${ }^{29}$

Fig. 5 gives the normalised frequency distributions for width dependence for all five groups. It is evident that the width dependence distribution for amblyopic eyes lies to the right, whereas the one for cataractous eyes lies to the left, with the other distributions somewhere between. There is a considerable overlap.

The result of the analysis of variance revealed a statistically significant difference between the groups: $F(4,175)=21 \cdot 0 ; p<0 \cdot 0001$. This suggests that the slope can also be used for differentiating between amblyopia and the other groups. Tukey's multiple range test indicated a significant difference (using $\alpha=$ 0.05 ) between the amblyopic group of eyes and all other groups.

\section{CONTRAST SENSITIVITY FOR THE LOW SPATIAL FREQUENCY} RANGE

The two differences already discussed were between the amblyopia group and the other groups. A third clear difference can be found between both optic nerve degeneration subgroups and the other groups of eyes. Fig. 6 gives the normalised distributions of the contrast sensitivity for the low spatial frequency range (CS(LF), calculated as the mean of the CSs for $0 \cdot 1,0 \cdot 2$, and $0.4 \mathrm{c} / \mathrm{deg}$ ) for the five groups. It is clear that the distributions for normal and amblyopic eyes do not greatly differ, while the optic nerve degener- 

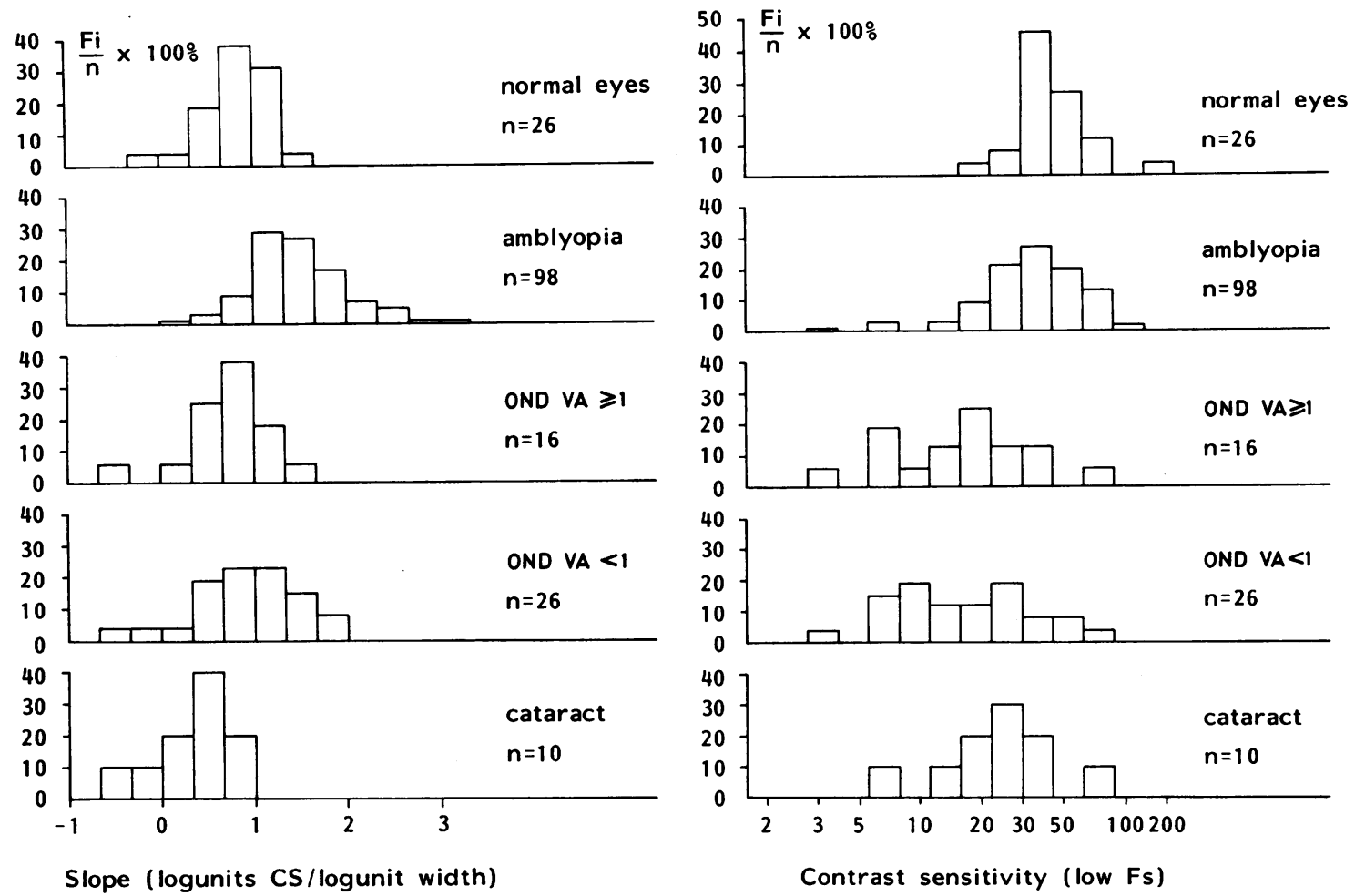

Slope (logunits CS/logunit width)

Fig. 5 Normalised frequency distribution for the width dependence of contrast sensitivity; further as Fig. 4.

ation subgroups in general have lower values, though there is a certain overlap with the other groups. It is a common finding that some cases of optic neuritis or multiple sclerosis show decreased CS for only low, middle, or high spatial frequencies, or for combinations. ${ }^{4510}$ The cataract group is somewhere in between; the distributions in Fig. 6 show that the one for cataract is not bimodal and so does not support the two-type classification of Hess and Woo. ${ }^{13}$ Inspection of the individual CSFs of cataractous eyes shows that low CS(LF) in this group may be due to the fact that in a considerable number of cases the CS(HF) was decreased to such an extent that not only the high frequency range but also the low frequency range was affected. Further, for the group of amblyopic eyes we did not find a bimodal distribution of CS(LF), as Hess and Howell postulated. ${ }^{2}$

The analysis of variance indicates a significant difference between the groups: $F(4,175)=16 \cdot 2$; $\mathrm{p}<0.0001$. The fact that the optic nerve degeneration subgroups with both normal and low VA had decreased CS(LF) (Fig. 2) is an indication that this difference is typical of optic nerve degeneration, and hence can be used in the differential diagnosis between amblyopia and optic nerve degeneration.

Fig. 6 Normalised frequency distribution for contrast sensitivity at low spatial frequencies; further as Fig. 4.

Tukey's multiple range test not only showed significant differences (using $\alpha=0.05$ ) between both optic nerve degenerative subgroups on the one hand and the groups with amblyopic and normal eyes on the other, but also that the CS(LF) for cataractous eyes was significantly lower than for normal eyes.

\section{COMBINATION OF FINDINGS}

Each of the three variables so far described can be used to differentiate between different visual defects. We investigated the diagnostic value of combining the three variables in one test to differentiate between some of the groups, as stated in the introduction: (1) between amblyopia and optic nerve degeneration with low visual acuity; and (2) between normal eyes and optic nerve degeneration with normal vision. We used logistic discriminant analysis $^{28}$ to answer the two questions.

Diagnosis on the basis of our chosen variables was compared with the known, correct diagnosis (as determined by a complete examination). The results for the differentiation between amblyopia and low vision optic nerve degeneration are depicted in Fig. 7. It shows the ROC curve, which is the relationship between the percentage of correctly diagnosed 


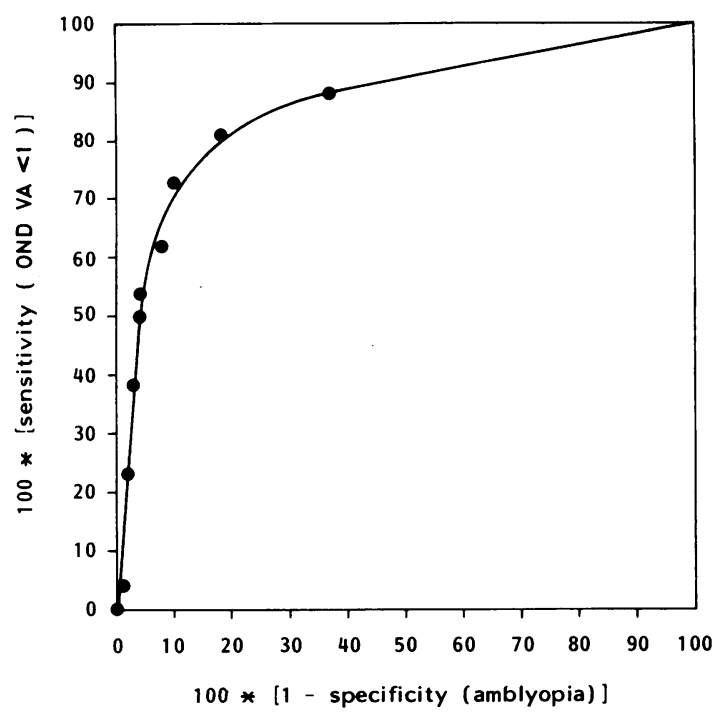

Fig. 7 ROC curve showing, for the combination of the three variables, the relationship between the diagnostic sensitivity for optic nerve degeneration with low visual acuity and the diagnostic specificity for amblyopia.

optic nerve degeneration cases (sensitivity of the tests), and the percentage of incorrectly diagnosed amblyopia cases (1-specificity). The curve shows that if one chooses limits which ensure that the sensitivity is high (that is, many cases of optic nerve degeneration are diagnosed correctly) specificity is

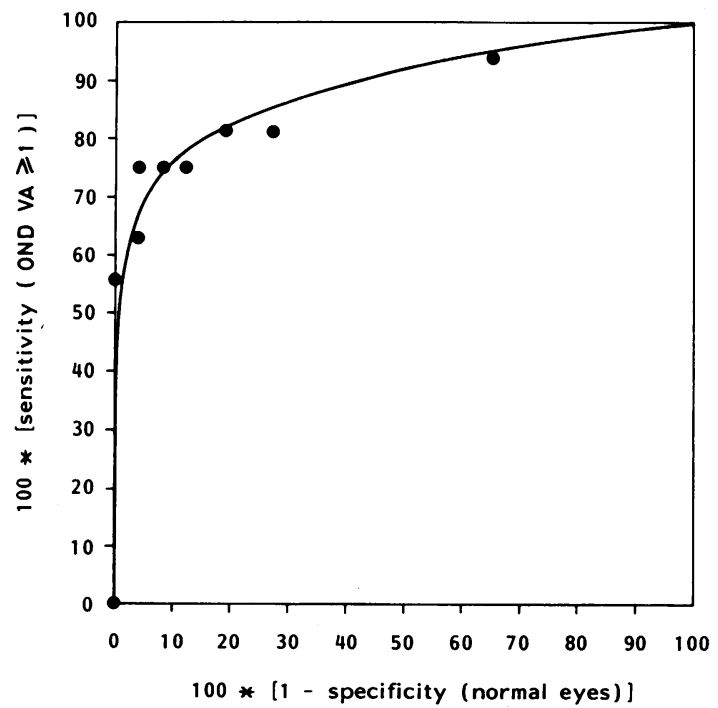

Fig. 8 ROC curve showing, for the combination of the three variables, the relationship between the diagnostic sensitivity for optic nerve degeneration with normal visual acuity and the diagnostic specificity for normal eyes.
Table 1 Results of logistic discriminant analysis for the group of amblyopic eyes and for the low vision optic nerve degeneration (OND) subgroup for a specific choice (see text)

\begin{tabular}{llll}
\hline \multirow{2}{*}{$\begin{array}{l}\text { Diagnosis resulting } \\
\text { from tests }\end{array}$} & \multicolumn{3}{l}{ Known diagnosis } \\
\cline { 2 - 4 } & OND $($ VA<1) & Amblyopia & Total \\
\hline OND & 21 & 18 & 39 \\
Amblyopia & 5 & 80 & 85 \\
Total & 26 & 98 & 124 \\
\hline
\end{tabular}

low (that is, many amblyopia cases are erroneously classified as optic nerve degeneration).

The clinician has to decide whether it is acceptable to miss a number of amblyopia cases in order to obtain correct diagnosis for a greater proportion of optic nerve degeneration cases. Table 1 gives one example of such a choice, in which both the sensitivity and the specificity are about $80 \%$. This choice corresponds to the following linear combination of the three variables discussed above:

$$
\mathrm{Z}=6 \cdot 7+0 \cdot 05^{*}(\mathrm{VA}-\mathrm{GA})-0 \cdot 18^{*} \mathrm{CS}(\mathrm{LF})-0 \cdot 4^{*} \mathrm{~S} \text {. }
$$

Whenever $\mathrm{Z}>-1.4$ the patient is assigned to the optic nerve degeneration group, otherwise to the amblyopia group. By means of this choice it turns out that a correct diagnosis is made in $80 / 98$ or $82 \%$ of the amblyopes and in $21 / 26(81 \%)$ of the optic nerve degeneration group, while an erroneous decision was taken in 23 out of 124 cases (19\%). The predictive value of the test for amblyopia is high: $80 / 85$ or $94 \%$. For optic nerve degeneration the predictive value is considerably lower: $21 / 39$ or $54 \%$. Of course these last values depend on the incidences of the two types of disorders in the tested population.

In a similar way results for the comparison between the optic nerve degeneration subgroup which has normal VA and the group of normal eyes are given in Fig. 8 (ROC curve) and Table 2 (result of logistic discriminant analysis). If limits for normal tests are chosen so that $95 \%$ of the 26 normal eyes can be considered as such, it turns out that 12 out of 16 cases of optic nerve degeneration with normal vision $(75 \%)$ are not assigned to the normal group despite the normal VA.

Table 2 Results of logistic discriminant analysis for the group of normal eyes and for the optic nerve degeneration (OND) subgroup with normal visual acuity for a specific choice (see text)

\begin{tabular}{llll}
\hline Diagnosis resulting & \multicolumn{3}{l}{ Known diagnosis } \\
\cline { 2 - 4 } & OND $(V A \geqslant 1)$ & Normal eyes & Total \\
\hline OND & 12 & 1 & 13 \\
Normal eyes & 4 & 25 & 29 \\
Total & 16 & 26 & 42 \\
\hline
\end{tabular}




\section{CONCLUSIONS}

In the introduction several questions were raised which we can now answer.

The shape of the spatial contrast sensitivity function is partly related to the type of pathology. Specific for amblyopia is a discrepancy between visual acuity and grating acuity (VA $<\mathrm{GA})$, in addition there is a strong dependence of contrast sensitivity on the width of the stimulus. In optic nerve degeneration there is a decreased contrast sensitivity in the low spatial frequency range; this happens in equal amounts in cases with decreased and with normal visual acuity. It is also found in a part of the group of cataractous eyes.

Decreased contrast sensitivity in the higher spatial frequency range occurs in all kinds of disorders of the visual system and is associated with reduced visual acuity (subject to the limitations expressed above for the group of amblyopic eyes).

The differences between the groups give valuable tests for the differentiation not only between amblyopia and optic nerve degeneration associated with reduced visual acuity, but also between optic nerve degeneration with normal visual acuity and normal eyes.

\section{References}

1 Campbell FW. Why do we measure contrast sensitivity? Behav Brain Res 1983; 10: 87-97.

2 Hess RF, Howell ER. The threshold contrast sensitivity function in strabismic amblyopia: evidence for a two type classification. Vision Res 1977; 17: 1049-55.

3 Hess RF, Campbell FW, Greenhalgh T. On the nature of the neural abnormality in human amblyopia; neural aberrations and neural sensitivity loss. Pflugers Arch 1978; 377: 201-7.

4 Zimmern RL, Campbell FW, Wilkinson IMS. Subtle disturbances of vision after optic neuritis elicited by studying contrast sensitivity. J Neurol Neurosurg Psychiatry 1979; 42: 407-12.

5 Hess RF. Contrast vision and optic neuritis: neural blurring. $J$ Neurol Neurosurg Psychiatry 1983; 46: 1023-30.

6 Harwerth RS, Levi DM. A sensory mechanism for amblyopia: psychophysical studies. Am J Optom Physiol Opt 1978; 55: 151-62.

7 Levi DM, Harwerth RS. Contrast sensitivity in amblyopia due to stimulus deprivation. Br J Ophthalmol 1980; 64: 15-20.

8 Bradley A, Freeman RD. Contrast sensitivity in anisometropic amblyopia. Invest Ophthalmol Vis Sci 1981; 21: 467-76.
9 Hagemans KH, van der Wildt GJ. The influence of the stimulus width on the contrast sensitivity function in amblyopia. Invest Ophthalmol Vis Sci 1979; 18: 842-7.

10 Regan D, Silver R, Murray TJ. Visual acuity and contrast sensitivity in multiple sclerosis-hidden visual loss; an auxiliary diagnostic test. Brain 1977; 100: 563-79.

11 Frisén L, Sjöstrand J. Contrast sensitivity in optic neuritis; a preliminary report. Doc Ophthalmol Proc Ser 1979; 17: 165-73.

12 Hyvärinen L, Laurinen P, Rovamo J. Contrast sensitivity in evaluation of visual impairment due to macular degeneration and optic nerve lesions. Acta Ophthalmol (Kbh) 1983; 61: 161-70.

13 Hess R, Woo G. Vision through cataracts. Invest Ophthalmol Vis Sci 1978; 17: 428-35.

14 Von Békésy G. A new audiometer. Acta Otolaryngol (Stockh) 1947; 35: 411-22.

15 Campbell FW, Robson JG. Application of Fourier analysis to the visibility of gratings. $J$ Physiol (Lond) 1968; 197: 551-66.

16 Hoekstra J, van der Goot DPJ, van den Brink G, Bilsen FA. The influence of the number of cycles upon the visual contrast threshold for spatial sine wave patterns. Vision Res 1974; 14: 365-8.

17 McCann JJ, Savoy RL, Hall JA. Scarpetti JJ. Visibility of luminance gradients. Vision Res 1974; 14: 917-27.

18 Van der Wildt GJ, Keemink CJ, van den Brink G. Gradient detection and contrast transfer by the human eye. Vision Res 1976; 16: 1047-53.

19 Keemink CJ, van der Wildt GJ, van Deursen JBP. Microprocessor controlled contrast sensitivity measurements. Med Biol Eng Comput 1979; 17: 371-8.

20 Bodis-Wollner I. Visual acuity and contrast sensitivity in patients with cerebral lesions. Science 1972; 178: 769-77.

21 Levi DM, Klein S. Differences in Vernier discrimination for gratings between strabismic and anisometropic amblyopes. Invest Ophthalmol Vis Sci 1982; 23: 398-407.

22 Levi DM, Klein S. Hyperacuity and amblyopia. Nature 1982; 298: 268-70.

23 Shapley RM, Tolhurst DJ. Edge detectors in human vision. $J$ Physiol (Lond) 1973; 229: 165-83.

24 Levi DM, Harwerth RS, Pass AF, Venverloh J. Edge sensitive mechanisms in humans with abnormal visual experience. Exp Brain Res 1981; 43: 270-80.

25 Stuart JA, Burian HM. A study of separation difficulty: its relationship to visual acuity in normal and amblyopic eyes. Am J Ophthalmol 1962; 53: 471-7.

26 Flom MC, Weymouth FW, Kahneman D. Visual resolution and contour interaction. J Opt Soc Am 1963; 53: 1026-32.

27 Dunn OJ, Clark VA. Applied statistics: analysis of variance and regression. New York: Wiley, 1974.

28 Lachenbruch PA. Discriminant analysis. New York: Hafner, 1975.

29 Van der Wildt GJ, Waarts RG. Contrast detection and its dependence on the presence of edges and lines in the stimulus field. Vision Res 1983; 23: 821-30.

Accepted for publication 24 April 1986. 